\title{
Research Paper \\ Multiple Relations Among Thought Control Strategies and Symptoms of Personality Disorders with Nightmare
}

\author{
Narjes Salimi Dehaghani ${ }^{1},{ }^{*}$ Zahra Yousefi²
}

\begin{abstract}
1. MA in Clinical Psychology, Department of Psychology, Faculty of Educational Sciences \& Psychology, Islamic Azad University, Khorasgan Branch, Isfahan, Iran. 2. PhD in Clinical Psychology, Assistant Professor, Department of Psychology, Faculty of Educational Sciences \& Psychology, Islamic Azad University, Khorasgan Branch, Isfahan, Iran.
\end{abstract}

\begin{tabular}{|c|c|}
\hline $\begin{array}{l}\text { Use your device to scan } \\
\text { and read the article online }\end{array}$ & Chatt on: Salimi Dehaghani N. Yousefi Z. [Multiple Relations Among Thought Control Strategies and Symptoms of Per- \\
\hline Dinitip & $\begin{array}{l}\text { sonality Disorders with Nightmare (Persian)]. Iranian Journal of Psychiatry and Clinical Psychology. 2018; 24(3):298-309. } \\
\text { http://dx.doi.org/10.32598/ijpcp.24.3.298 }\end{array}$ \\
\hline 674st4 & dol': http://dx.doi.org/10.32598/ijpcp.24.3.298 \\
\hline
\end{tabular}

Funding: See Page 308

(c) Copyright: The Author(s)

Received: 25 Aug 2017

Accepted: 01 Jun 2018

Keywords:

Thought control strategies, Personality disorder, Nightmare

\section{A B STRACT}

Objectives Nightmare is one of the sleep problems among adolescents. This research aimed to examine multiple relations among thought control strategies and symptoms of personality disorders among female students in Shahreza City, Iran in 2015-2016.

Methods In this correlational study, the statistical population comprised all female students in pre-university schools and the sample was 300 girls that they were randomly selected from students list. The measurements were thought control strategies, Millon Clinical Multiaxial Inventory, and Nightmare Scale (2014). The obtained data were analyzed by Pearson correlation and stepwise regression in SPSS V. 21.

Results The results showed among thought control strategies, worry and among personality disorders, dependent and histrionic personality disorders have positive and significant relations with symptoms of nightmare but antisocial and narcissistic personality disorders have negative and significant relations with nightmare. Worry, histrionic, dependent and narcissistic personality disorders can predict nightmare.

Conclusion Based on the results, changes in worries, dependent, histrionic and narcissistic and antisocial personality disorders can predict nightmares.

\section{Extended Abstract}

\section{Introduction}

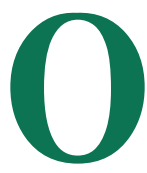

ne of the important components of physical and mental health is restful sleep. Some of brain processes occur in sleep that they are important in healthy function in overall physical and mental health. Research suggest that restful sleep help to provide new pathways of processing information that help to adequate function of memory, learning, information processing speed, creativity, problem solving and making decision. With disrobed sleep, physical changes happen in brain that they cause to change executive function of brain and overall of brain's function. Individual have not restful sleep may suffer from difficulty in making decision, problem solving, controlling emotions. Depression, suicide thought or risk-taking behavior are the other results of inadequate sleep.

One reason to disrupt of sleep is nightmare. Nightmares are dreams lengthy and stimulate anxiety, worry, and fear. All of people have little nightmares but repeated nightmares can be disorder if they associate with decreasing of occupational, social or other dimensions of daily life. As regard to effect of daily life in different dimensions on sleep, it seems

* Corresponding Author:

Zahra Yousefi, PhD

Address: Department of Psychology, Faculty of Educational Sciences \& Psychology, Islamic Azad University, Khorasgan Branch, Isfahan, Iran.

Tel: +98 (31) 35354001

E-mail: z.yousefi@khusif.ac 
thought control strategies can be associate with nightmares. Thought control strategies point out to method the people use to control intrusive thoughts that they are central components of emotional disorders such as obsessive-compulsive disorder, depression, post-traumatic disorder or generalized anxiety disorder. However, thought control strategies may play role in forming nightmare but personality is important feature of coping stress in daily life; as result it may play important role in forming nightmare.

Individual with personality disorder tolerate and impose more interpersonal and interpersonal because of their disorder. Their problems may appear with hidden face in their dreams in forming nightmare. In total, as regard to importance of restful sleep among adolescents, the goal of the research was to examine multi relations among thought control strategies and symptoms of personality disorders among female students in Shahreza in 2015-2016.

\section{Method}

The method was descriptive and correlational type. The statistical population was all female girls in pre-university and the sample was 300 girls that they were randomly selected from students list in each school in Shahreza based on Tabachnick and Fidell [19] perspective that it is necessary for each scale and subscale should select 15 subjects. The measurements were thought control strategies [10], Millon Clinical Multiaxial Inventory [22] and Nightmare Scale [20]. All the measurements include appropriate psychometric characters. Gathered data was analyzed to assessed research hypothesis (there are multiple relations among thought control strategies and personality disorders with nightmare symptoms among pre-university students) by descriptive statistic (means and standard deviations) and inferential statistic (Pearson correlation and stepwise regression). The calculation was done by SPSS V. 21.

\section{Results}

The results of Pearson correlation showed among thought control strategies, worry and among personality disorders, dependent and histrionic personality disorders have positive and significant relations with symptoms of nightmare but antisocial and narcissistic personality disorders have negative and significant relations with nightmare. The results of stepwise regression showed worry, histrionic, dependent and narcissistic personality disorders can predict nightmare.

\section{Discussion}

Regard to results, the results found evidence that they show relations between worry and some types of personality disorders therefore it can be said changes in worries, dependent, histrionic and narcissistic and antisocial personality disorders can predict nightmares. This research look like other research has some limitations that they root in the nature of correlational research. The result can merely predict a variable from another variable therefore we cannot make causal conclusions. Perhaps the study involved female students in shahreza city, thus, it may be uncertain whether the correlational findings may generalize to other people or situations. As regard results it can be said one way to improve nightmare symptoms is decreasing worries and another way is decreasing the symptoms of personality disorders.

\section{Ethical Considerations}

\section{Compliance with ethical guideline}

The research was adhered six points with subjects: informed consent, voluntary participation, not to harm, confidentiality, anonymity, only assessing relevant components.

\section{Funding}

The research had no financial support.

\section{Conflict of Interest}

The authors have no conflict of interest.

\section{Acknowledgements}

All the participants are appreciated for their cooperation. 


\title{
روابط جندكَانه بين راهبردهاى كنترل فكر و علائم اختلالات شخصيت با علائم رؤياهاى ترسناك
}

\author{
نرجس سليمى' "زهرا يوسفى
}

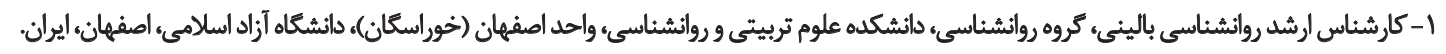

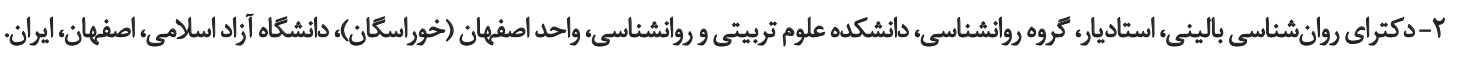

\begin{abstract}
حكيد
تاريخ دريافت: ب شهريور

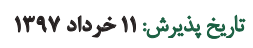

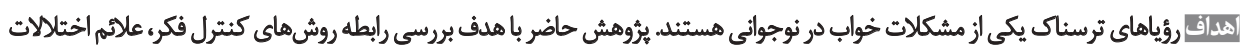

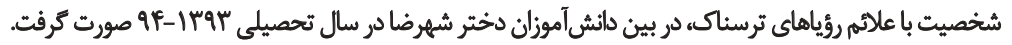

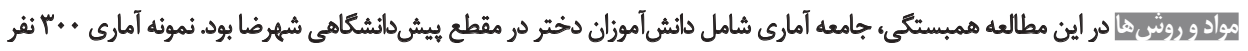

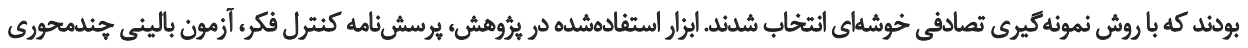

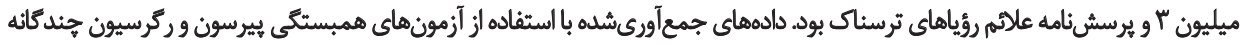

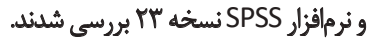

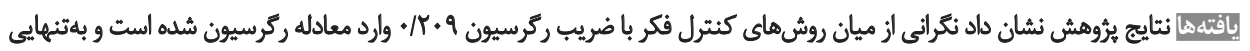

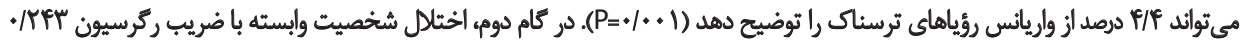

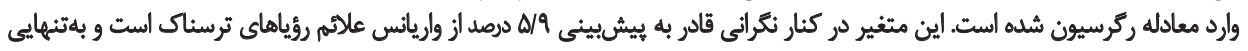

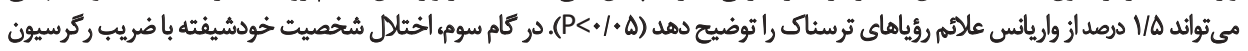

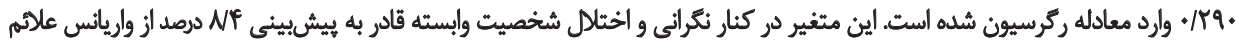

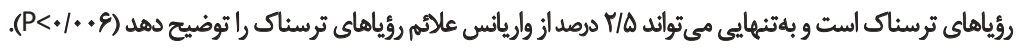

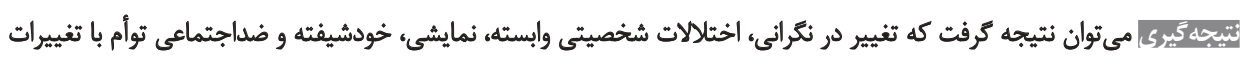

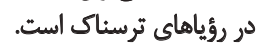

كليدوازثها:

روش شهاي كنترل فكر، اختيلال شخصيث، رؤياهاى كنتري فيري ترسيناك

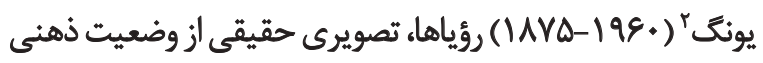

مقدمه

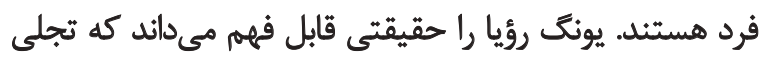

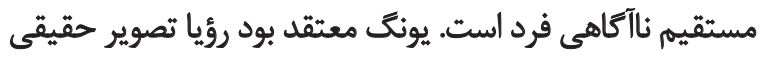

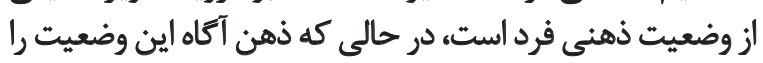
انكار مى كند و يا با آن در تضاد است دريت

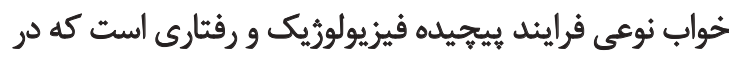

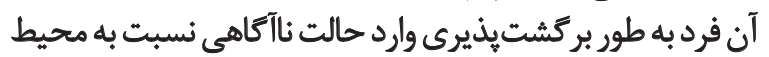

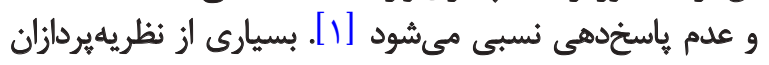

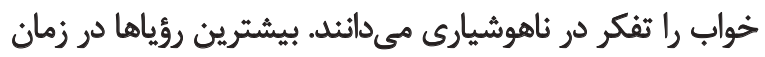

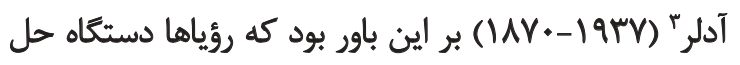

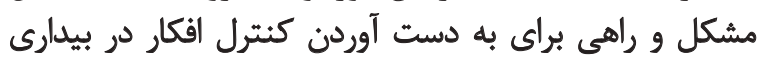

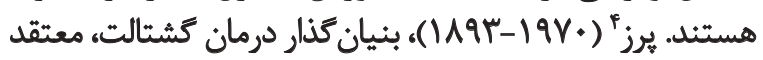

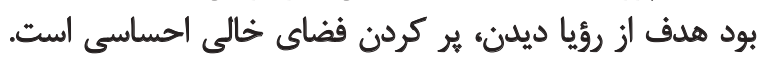

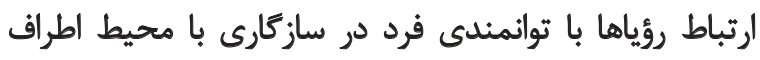

\section{Jung}

3. Adler

4. Perls

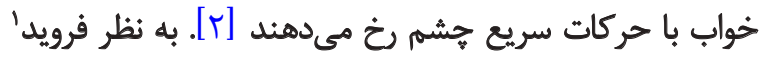

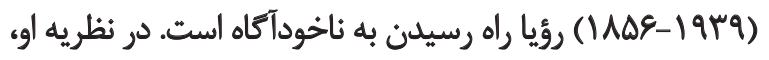

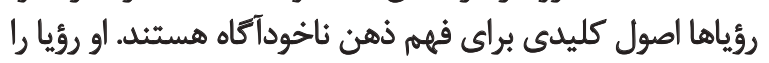

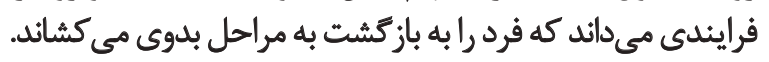

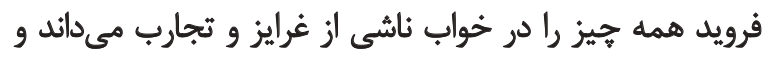

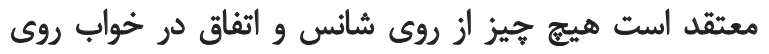

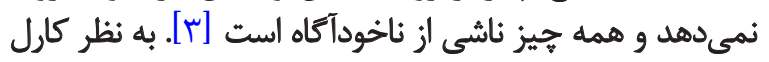

1. Freud
\end{abstract}

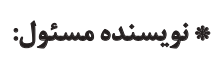

دكتر زهرا يوسفى نوين

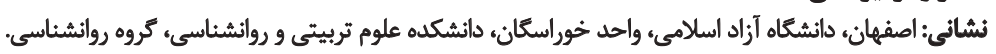

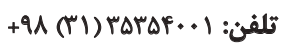
يست الكترونيكي: تيف: z.yousefi@khusif.ac 


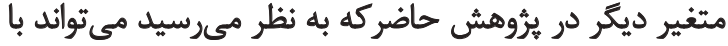

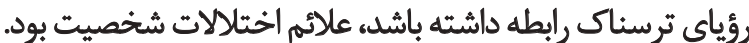

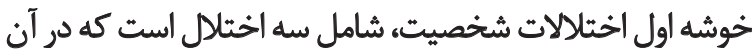

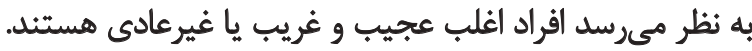

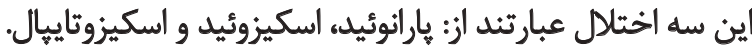

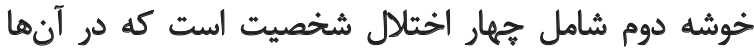

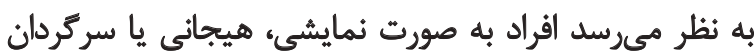

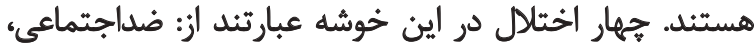

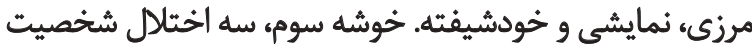

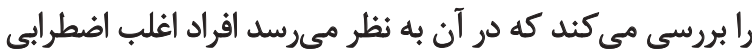

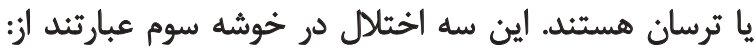

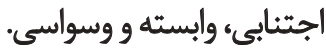

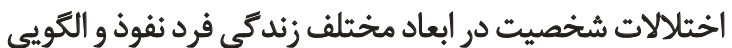

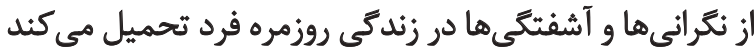

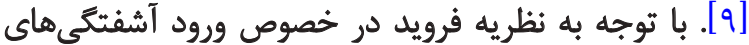

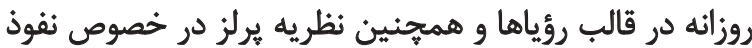

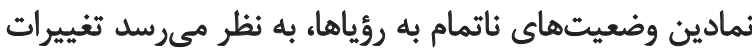

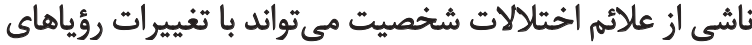

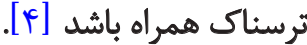

با توجه به دوران حساس نوجواني و بادر نظر كرفتن آمار بالاى

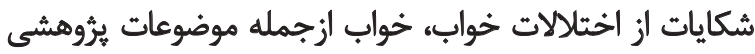

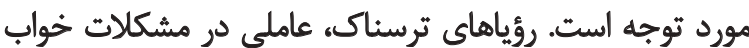

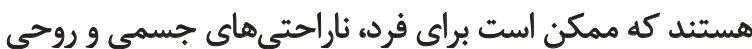

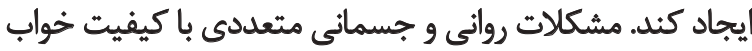

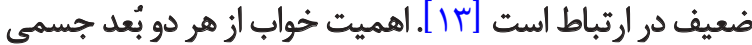

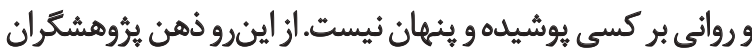
همواره دريير عوامل مرتبط با مشكلات خواب بوده است. ازجمله ضرورتهاى بررسى اختلال خواب و كابوس هاو رؤياهاى

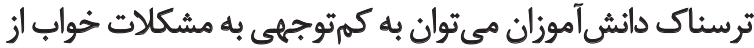

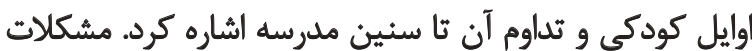

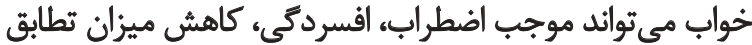

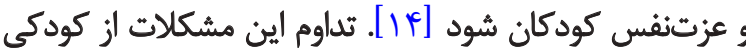

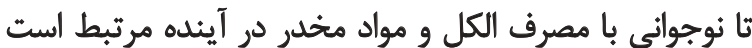

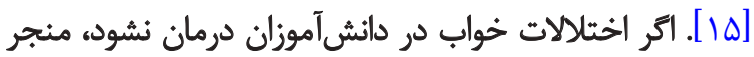
به عوارضى نظير اختال در توجه و تمركز، حافظه و و يادكيرى و

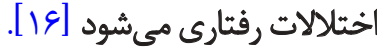

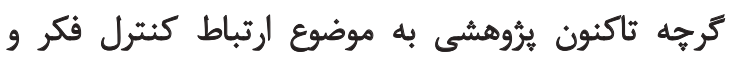

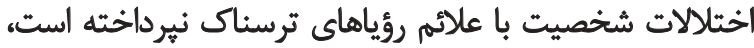

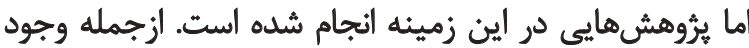

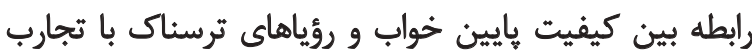

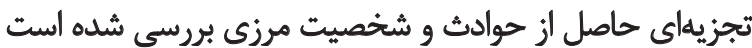

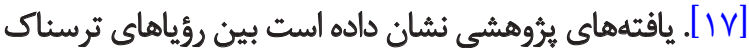

موضوع جالب توجهى است؛ هجون نكَرانى و تشويش حاصل از

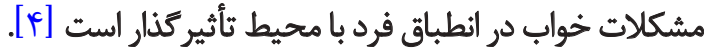

در دوران نوجوانى تغييرات متداول بيولوزيكي، روانشناختى و

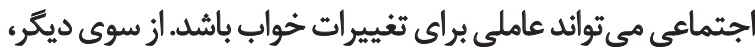

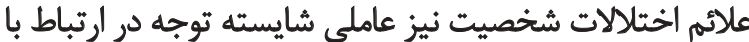

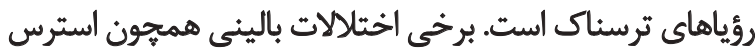

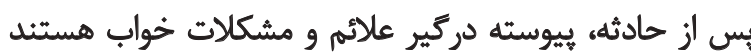

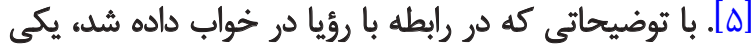

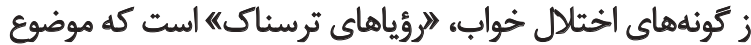

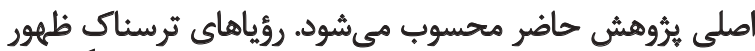

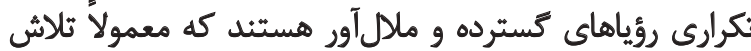

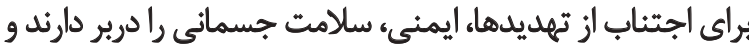

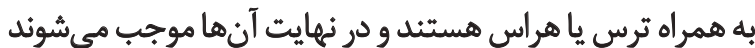
شخص از خواب بيدار شود و در حالت هشيارى قرار كيرد رؤياهاى ثرسناك ازجمله خوابيريشىهاى هائي رائى هستند

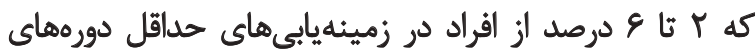

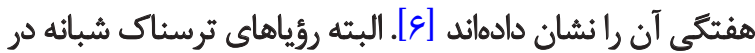

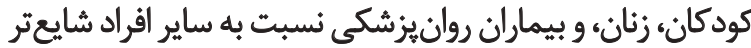

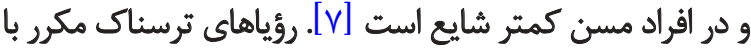

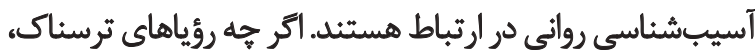

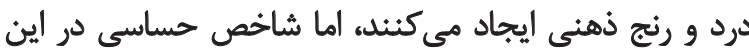

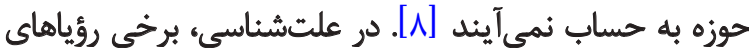

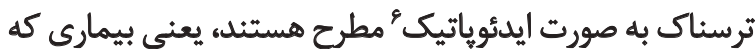

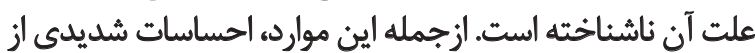

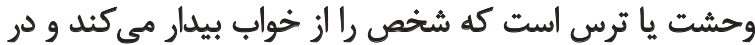

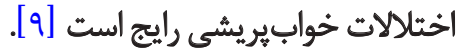

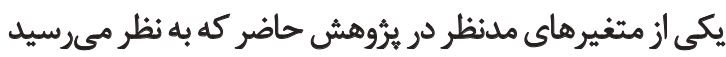

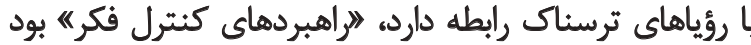

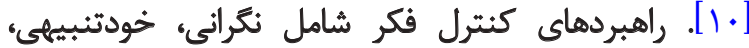

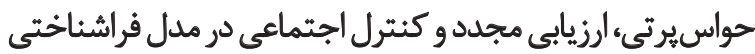

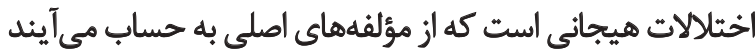

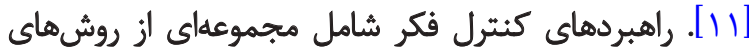

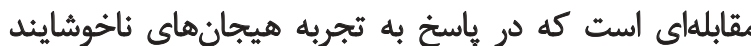

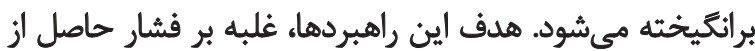

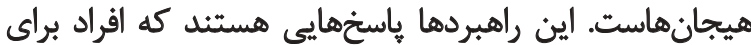

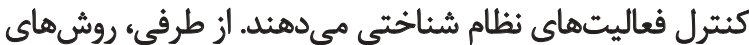

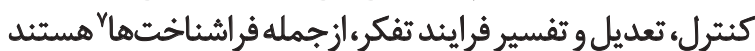

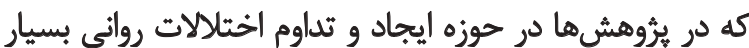

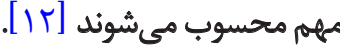

5. Parasomnia

6. Idiopathic

7. Metacognition 


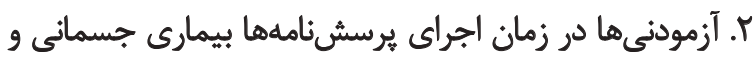

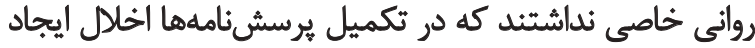

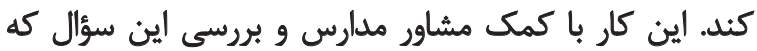

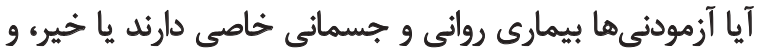

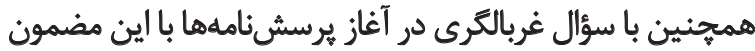

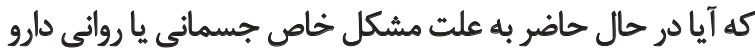

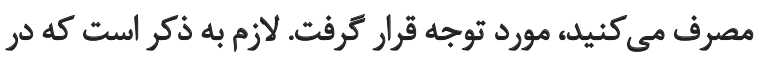

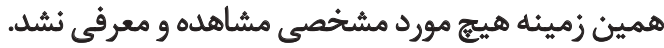

در اين يُروهش از سه يرسشنامه استفاده شد. ضمن اينكه

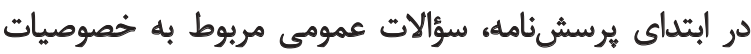

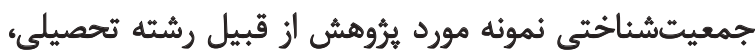
معدل، درآمده شغل بدر و.... قرار داده شد. بند.

\section{برسش نامه علائم وؤياهاى ثرسناك}

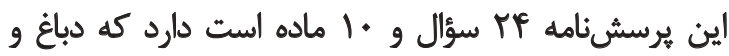

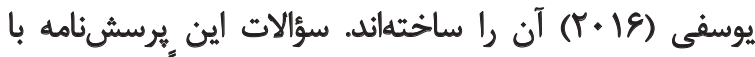

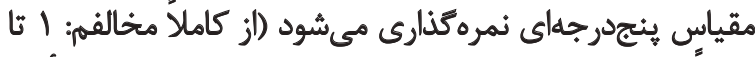

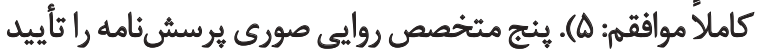

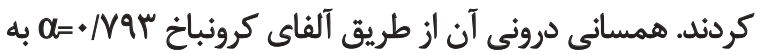

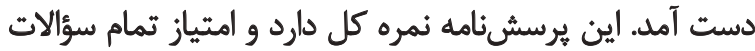

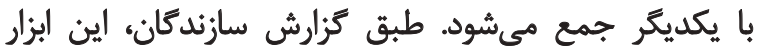

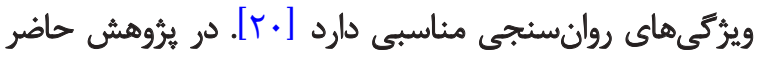

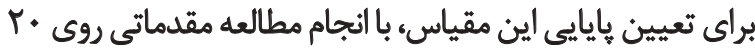

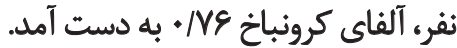

$$
\text { مبوسشنامه كثترل فكر ولز و ديويس }
$$

اين يرسشنامه • ب سؤالى و ه زيرمقياس دارد كه عبارتند ازي: 1.

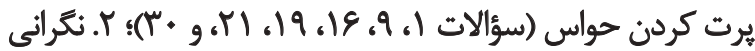

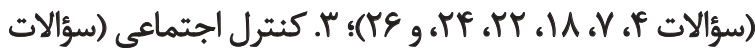

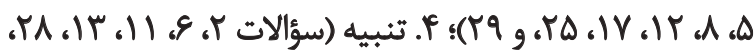

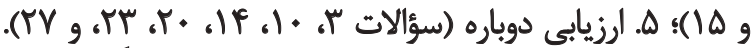

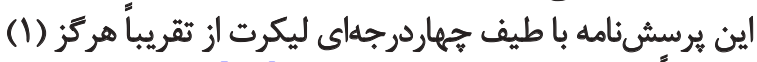

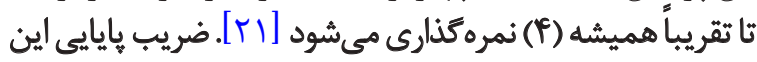

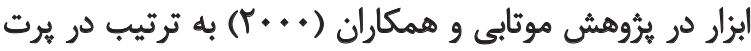

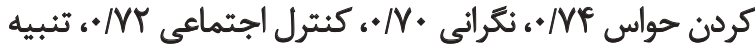

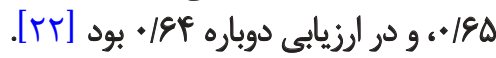

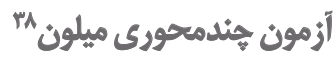

آزمون جندمحورى ميلون نوعى مقياس خودسنجى است كه

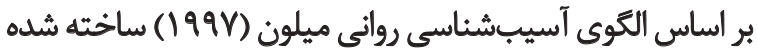
است. اين آزمون براى تصميمكيرى بالينى و تشخيص ابتلاي

8. Millon Clinical Multiaxial Inventory
با نشخوار فكرى و خُلق بايين رابطه معنادار وجود دارد [1 ا]. با در نظر داشتن اهميت خواب آرام در رشد همهجانبه افراد

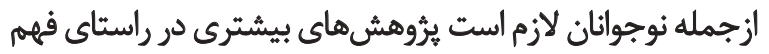

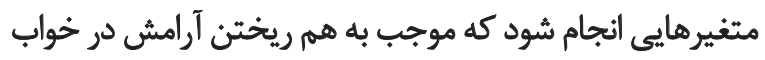

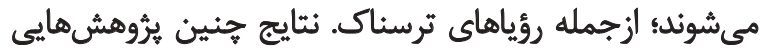

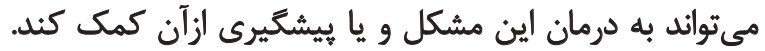

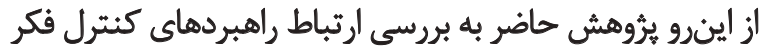

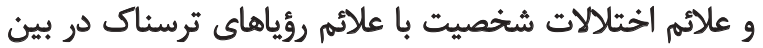
دانشآموزان دختر مقطع دبيرستان شهرستان شهرضا عاديا در سال

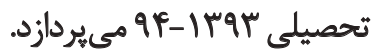

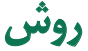

تحقيق حاضر جزء مطالعات توصيفى و از نوع همبستكى دماري

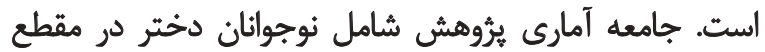

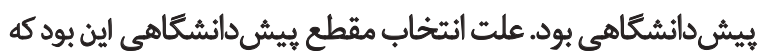

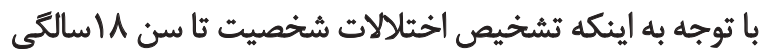

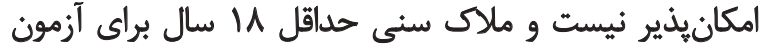

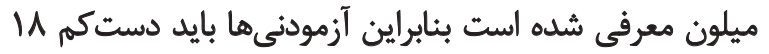

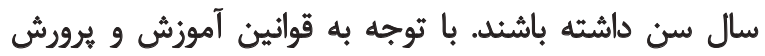

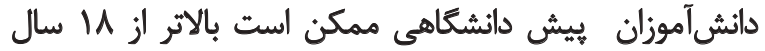

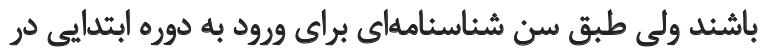
بيشدبستانى حداقل 1/ إل سال دارند.

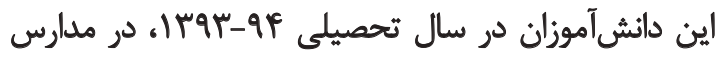
شهرضا تحصيل مىكردند كه تعداد آنها

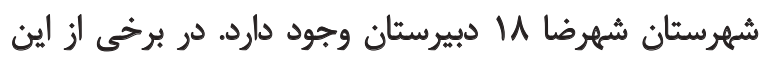

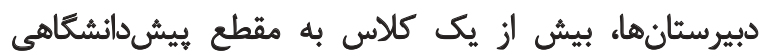

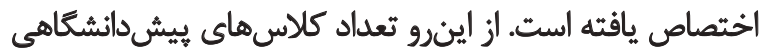

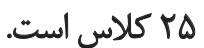

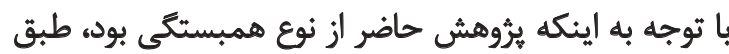

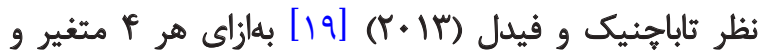

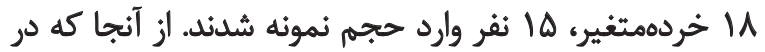

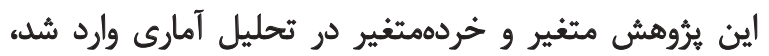

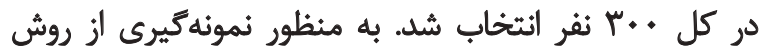

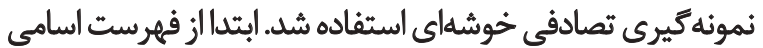

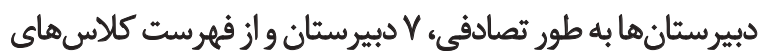

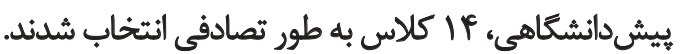
معيارهاى ورود به مطالعه اين موارد بود: ا. با توجه به اينكه

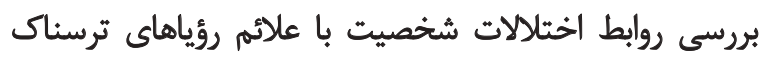

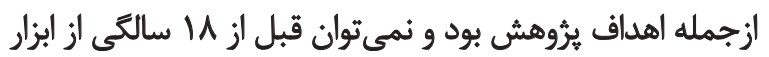

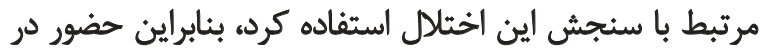

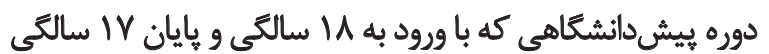

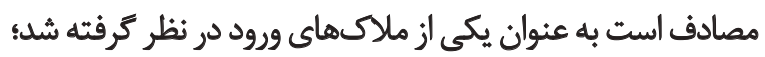


يُؤهش براي اولياى هر هدرسه و جلب توافق آنان و دريافت

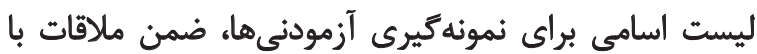

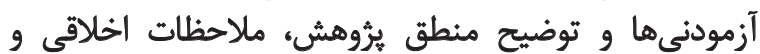

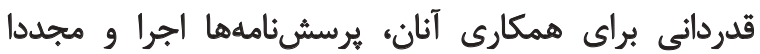

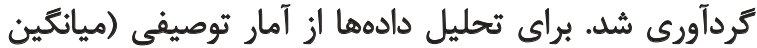

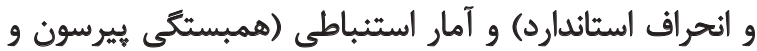

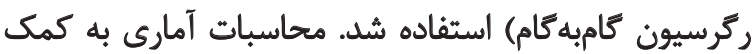

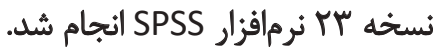

يأتنهها

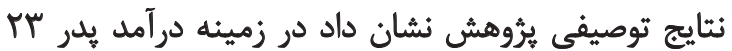

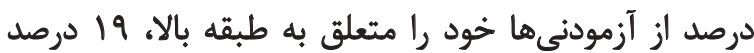

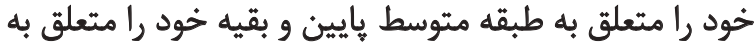

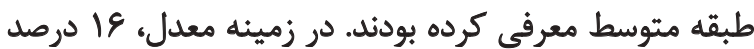

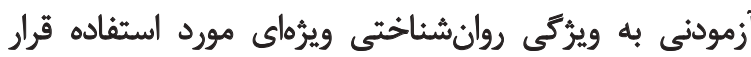

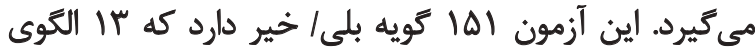

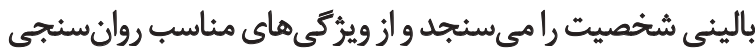

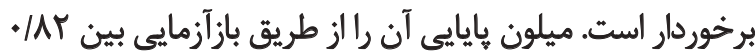

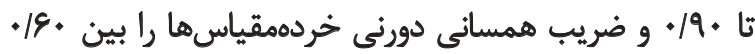

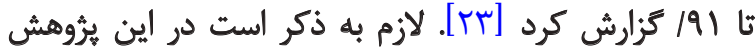

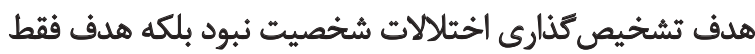

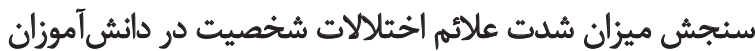
و بررسى ارتباط اين شدت با علائم روياهاى ترسناك بودات

$$
\text { روش الجرا }
$$

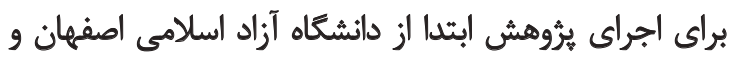

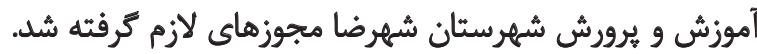

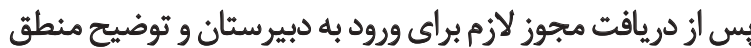

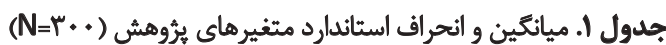

\begin{tabular}{|c|c|}
\hline ميانكين (انحراف استاندارد) & متغيرها \\
\hline$\Delta N W(I D / A V)$ & رؤياهاى ترسناك \\
\hline$|E| \cdot r(\varphi / \cdot A)$ & يرت كردن حواس \\
\hline V/AN(T/PI) & ن اتكرانى \\
\hline $\mid r / e q(Y /+\varphi)$ & كنترل الجتماعى \\
\hline $11 / \Delta \cdot(T / Q)$ & تنبيه \\
\hline$|r / F|(r /+V)$ & ارزيابى دوباره \\
\hline$I E / Y P(V / N T)$ & اسكيزوئيد \\
\hline ID/ED(I/TA) & 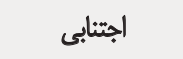 \\
\hline $\operatorname{IV/FO}(1 / 19)$ & وابسته \\
\hline$W / \Delta(M / P \cdot)$ & نمايشى \\
\hline$r Y N a(Y / \Delta V)$ & حخودشيقته \\
\hline $\mid r / q \&(\cdot / R q)$ & ضداجتماعى \\
\hline $19 / \mu T(1 / M P)$ & آزاركر \\
\hline WEF $(Y / \cdot Y)$ & 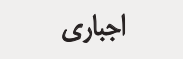 \\
\hline $\mid E / \Delta N(\cdot / N E)$ & منفى مرا \\
\hline $10 / r \Delta(Y /+9)$ & خودناكامساز \\
\hline $\mathbb{H} / q f(* / M T)$ & مرزى \\
\hline $\mid Q / 9 Y(\cdot M)$ & يارازوتيد \\
\hline $1 \otimes / 1 T(\cdot / \Delta \cdot)$ & اسكيزوتاييى \\
\hline
\end{tabular}


جدول rا. ماتريس همبستّكى ييرسون بين متغيرهاى يُيشبين و ملاك

\begin{tabular}{|c|c|c|c|c|c|c|c|c|c|c|}
\hline نمايشب & وابسته & إجنابى & السكيزوئيد & ارزيابى & تنييه & اجتماعي & تئرانى & هرت هرون & ترؤياهاى & متغيرها \\
\hline +/lfe" & o/ & .1 .98 & .1 .81 & 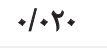 & $+1 \cdot v e$ & 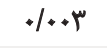 & $.1190^{\circ}$ & .1 .88 & 1 & رؤياهاي ترسناك \\
\hline 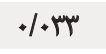 & $-+/ 1+)^{*}$ & $.1 .8 r$ &.$- / * \mathrm{~V}$ &.$/ 411 *$ & . /Wer &.$/ 119^{* *}$ & $\cdot 1 / \cdot \cdot 9$ & 1 & & يرت كردن حواس \\
\hline .1 .98 & $+1+\Delta F$ & $-* 1 \cdot+\varphi$ & $+M \mathrm{M}^{* *}$ &.$(M Y)^{*}$ &.$/ R V \Delta^{*}$ &.$/ 110^{\circ}$ & 1 & & & نكرائي \\
\hline $.1 \cdot r A$ & .1 .84 &.$/ 0 A r$ &.$- / . \Delta r$ & . & $.1+19$ & 1 & & & & كنترل اجتماعى \\
\hline$+/ N T^{*}$ & H.rT & - MreE & $.1 .9 \gamma^{* *}$ & $+/ 4 \cdot e^{*}$ & 1 & & & & & تنييه \\
\hline$+/ M n I^{*}$ & $+1+r T$ & AHE & 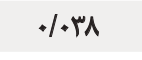 & 1 & & & & & & ارزيايى دوباره \\
\hline$. / 1 / 19^{*}$ &.$/ r \Delta Q^{\circ}$ & $\mathscr{H} \cdot M$ & 1 & & & & & & & اسكيزوئيد \\
\hline$\cdot / r \cdot \varphi *$ & $.1 \cdot \pi$ & 1 & & & & & & & & الجتنابي \\
\hline.$/ \pi r^{*}$ & 1 & & & & & & & & & وابسته \\
\hline \multirow[t]{10}{*}{1} & & & & & & & & & & نمايشى \\
\hline & & & & & & & & & & خوششيفته \\
\hline & & & & & & & & & & ضلاجتماعى \\
\hline & & & & & & & & & & آزآزير \\
\hline & & & & & & & & & & اجبارى \\
\hline & & & & & & & & & & منفقئر| \\
\hline & & & & & & & & & & خوناكامساز \\
\hline & & & & & & & & & & مرزى \\
\hline & & & & & & & & & & بارانوئيد \\
\hline & & & & & & & & & & اسكيزوتيييى \\
\hline
\end{tabular}

\begin{tabular}{|c|c|c|c|c|c|c|c|c|c|}
\hline اسكيزوتاييى & باراتوئيد & هرزى & خودناكامساز & منفى & الجبارى & آزارئ & ضداجتماعى & خودشيفته & مثنيرها \\
\hline.$/ * n$ & $\%$ &.$/ .+f$ & ..$Y \Delta$ & .1 .9 &.$/ * A$ & $.1 \cdot 14$ &.$- / \cdot n * *$ & $-* / 1 \ldots * *$ & رؤياهاى تربسناك \\
\hline -./.r. & $-* / * \Delta$ &.$- / . \Delta T$ & -.1 .81 &.$- / P \Delta^{*}$ &.$- / 11 r^{* *}$ &.$- / 1$ ve* &.$/ .14$ & $-.1 .8 V$ & برت كردن حواس \\
\hline .1 .91 & $-.1+p q$ &.$- / . r A$ &.$/ . r A$ & . /Im &.$/ . \Delta V$ & .1 .88 & $-.1+18$ & .1 .18 & نكرائى \\
\hline.$/ .14$ & -.1 .10 & -.1 .81 & $\cdot|\cdot|+\mid$ & $.1 . .9$ & $-.1 .98 * *$ & 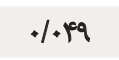 & $-.1 \cdot r$ &.$/ \cdot r r$ & كتترل اجتماعى \\
\hline.$/|Q|^{*}$ & $-* / * 18$ &.$/ .49$ & $\cdot M \cdot P *$ & $1 / 1$ & $-* 1+f r$ & $.1+\mathrm{PV}$ &.$/ . r$ & $. / 1 m \pi=*$ & تنبيه \\
\hline $.1 \cdot V A$ & -.1 .80 &.- .1 .49 & $-* / \cdot A r$ & $-. .1 .+9$ &.$- / . M r$ & .1 .81 &.$-|+r|$ & $.1 \cdot p r$ & ارزيابي دوباره \\
\hline . MUAH* &.$/ M n^{*}$ & . $/ Y *^{*}$ & $.1 .9 r$ & . MAY" &.$/ m W^{*}$ &.$/ 10^{\circ}$ &.$/ T V \cdot$ &.$/ M T V^{*}$ & السكيزوئيد \\
\hline$\cdot / \cdot A V$ &.$/ 419^{*}$ & $\cdot / r \cdot g^{*}$ & $\cdot / \Lambda \cdot \Lambda^{*}$ & $\cdot / r \cdot 9^{*}$ &.$/\left.M\right|^{e *}$ & . $/ . \Delta Y$ & . Mife & $.1 \Delta 59^{\circ}$ & اجتثنابى \\
\hline.$/ M T A^{*}$ &.$|18|^{*}$ &.$/ \% \omega^{*}$ & $\cdot 1 \cdot \Delta f^{e}$ &.$/ T V \Delta^{\circ}$ & $\cdot / \pi^{\prime} \cdot *$ & $\cdot / M^{\prime \prime} \cdot \Delta^{*}$ & - Mrepe* &.$/ T A_{*}^{*}$ & وابسته \\
\hline.$/ M E V^{*}$ &.$/ 1 \Delta \Delta^{*}$ & $\cdot / \mu+4 *$ & $.1 . r \Delta$ &.$/ \pi m$ & $. / M m=*$ &.$/ 480^{\circ}$ &.$/ T \Delta \sigma^{*}$ & $\cdot / \mu \cdot)^{*}$ & نمايشى \\
\hline.$/ M \mu Q^{*}$ & •/YAI* & $\cdot \pi+9^{*}$ & */ATI* & ./HTI* & ./NF" &.$/ M 9 Q^{*}$ & / & 1 & خونشيفته \\
\hline$+/ r+9^{*}$ & $+/ F \mid r$ & .Mqe* & ./IFE: &.$/ 490^{\circ}$ & -larf* & $\cdot / M+\Lambda^{*}$ & 1 & & ضلاجتماعى \\
\hline (MTru* & $+/ M r$ & $\cdot / \mu \cdot \Lambda^{*}$ & $.10+1$ &.$/ F N^{*}$ & ./KEY* & 1 & & & آزازارك \\
\hline . Tra. &.$/ m 19^{*}$ &.$/ A V^{*}$ & ./NF"** & $\cdot \mathbb{N}^{*}+\mathrm{N}^{*}$ & 1 & & & & آجبارى \\
\hline$\cdot / r^{*} r^{*}$ & - MFfe" &.$/ \Delta \Delta \Lambda^{*}$ & $110^{\circ}$ & 1 & & & & & منفىكر| \\
\hline.$/ 4$ & $+/ \kappa q *$ &.$/ 189 *$ & 1 & & & & & & خودناكامساز \\
\hline$\cdot / r W^{*}$ & $\cdot / \mu r I^{*}$ & 1 & & & & & & & مرزى \\
\hline$\cdot / M A^{*}$ & 1 & & & & & & & & يارازؤيد \\
\hline 1 & & & & & & & & & السكيزوناييي \\
\hline
\end{tabular}


جدول ץ. تحليل ركرسيون به منظور بيشبينى علائم رؤياهاي ترسناك بر مبناي نثرانى و اختلالات شخصيت وابسته، خودشيفته، نمايشى و ضداجتماعى

\begin{tabular}{|c|c|c|c|c|c|c|c|c|}
\hline معنادارى & درجه آزادى & درجه آزادى & $F$ & غالصهم & مجذور ضريبون & رئرسيون & مثغيرهاى واردشده & كام \\
\hline $.1+.1$ & YAP & 1 & Ir/qVO & $+1+\mu$ & 1.ppr & $+/ r+9$ & نكراثيى & 1 \\
\hline זr./. & TAT & 1 & .104 & $.1 \cdot 10$ & $\% /+\Delta q$ & תוT/. & وابسته & $r$ \\
\hline $.1 .+8$ & rAT & 1 & V/MT & 1. TA &.$/ A f$ &.$/ \times q$. & خودشيفته & $r$ \\
\hline
\end{tabular}

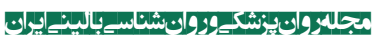

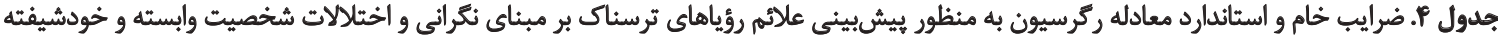

\begin{tabular}{|c|c|c|c|c|c|}
\hline معنادارى & $\mathrm{T}$ & ضريب بتا & خطاي الستاندارد & ضريب خام (B) & مثغير \\
\hline . &.$\left./ 9{ }^{\prime}\right)$ & - & IV/TFi & $\mid 8 / \cdot \% \Delta$ & مقدار ثابت \\
\hline $.1+1$ & $r / \Delta V Q$ &.$/ 144$ & . MEA & $1 / r+\varphi$ & نكرائي \\
\hline $.1 .+1$ & r/ETr & . & - Nar & r/ArV & وإبسته \\
\hline $.1 .+1$ & $-P / V \wedge q$ &,$- / m p p$ & - MTr & & خولشيفته \\
\hline
\end{tabular}

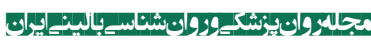

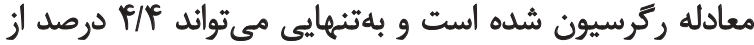

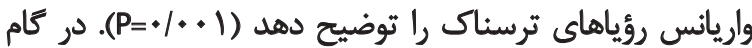

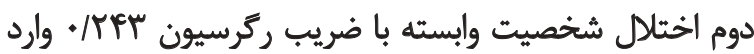

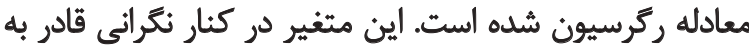

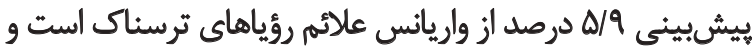

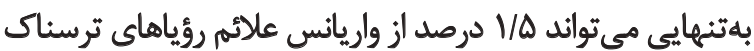

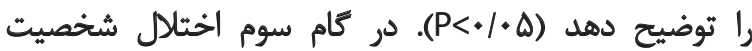

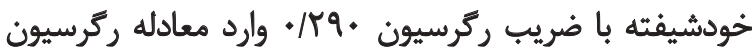

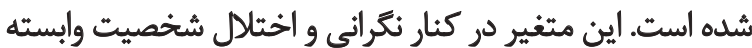

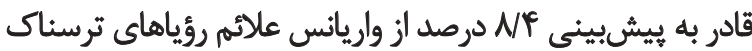

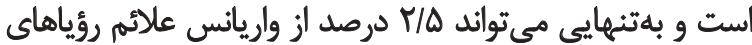

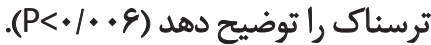

همانطور كه در جدول شماره \& ملاحظه مى مود، ضرايب خام

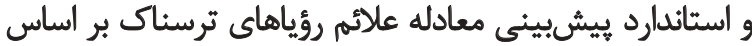

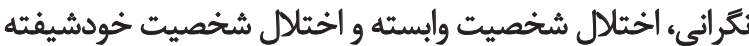

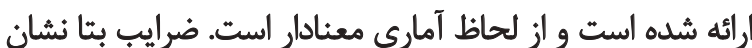

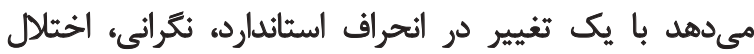

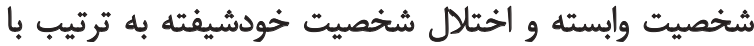

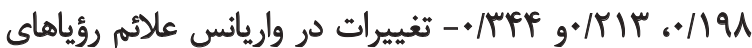

$$
\text { ترسناك همراه است. }
$$

بحث

نتايج يُوهش حاضر نشان داد از بين ابعاد كنترل فكر و

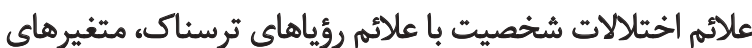

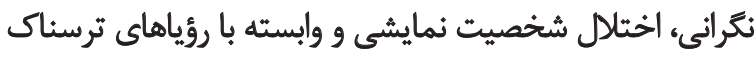

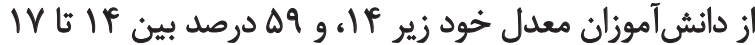

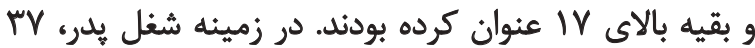

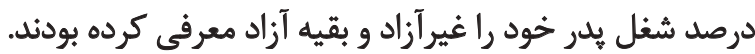

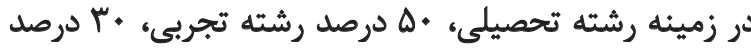
علوم انسانى و بقيه رياضى بودند.

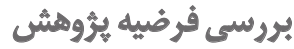

بين ابعاد كنترل فكر و علائم اختلالات شخصيت با علائم

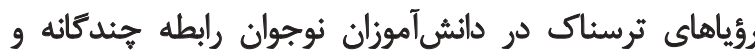

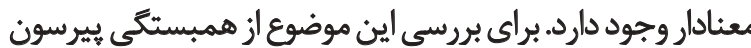

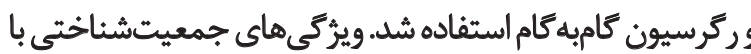

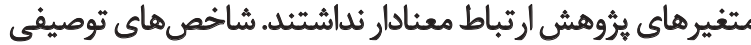

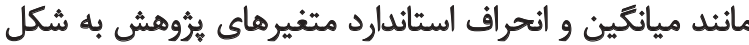
كلى در بين آزمودنىها در جدول شمانين اسنداره ما آمده است.

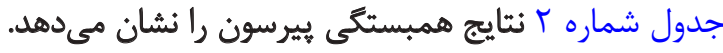

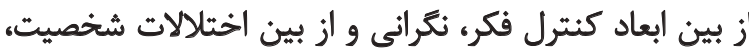

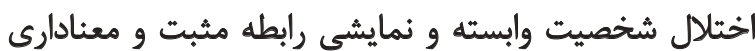

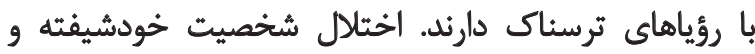

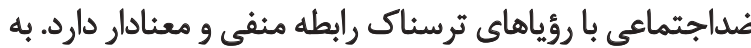

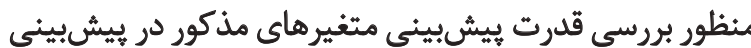

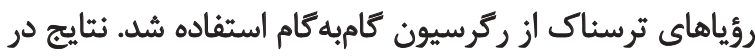
جدول شماره ب آمده است.

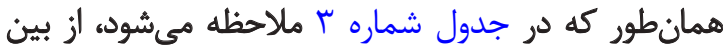

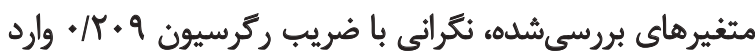


فراكير و بيش از حد براي دريافت مراقبت از سوى ديكًران همراه

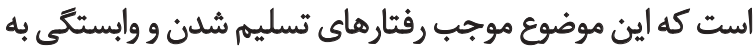

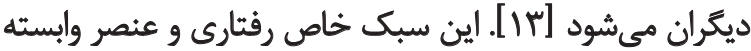

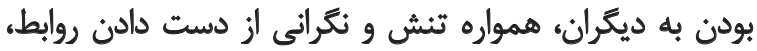

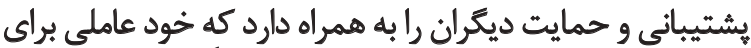

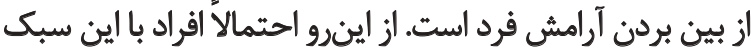

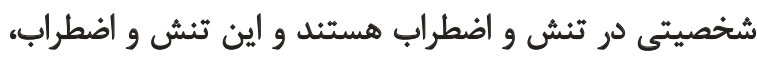

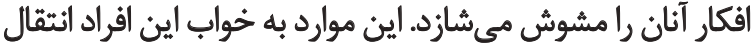

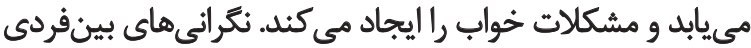

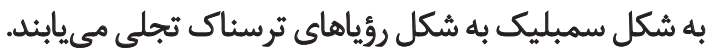

اين تبيين احتمالى در ارتباط اختلال شخصيت نمايشى و رؤياهاى ترسناك نيز صادق است. اين نوع ازين اختين اختلال شخصيت،

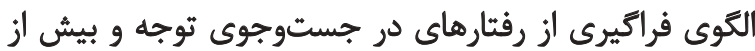

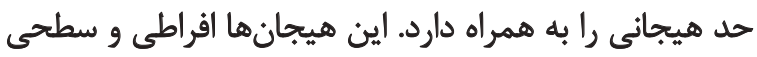

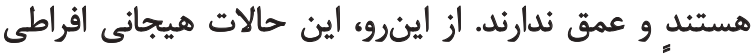

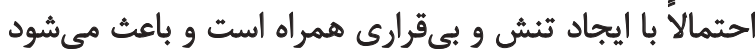

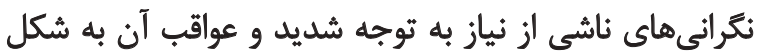

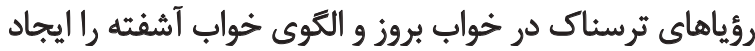

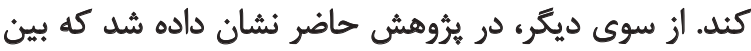

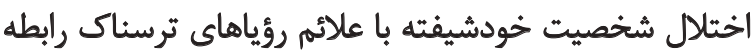
منفى و معنادارى وجود دارد.

با توجه به اينكه شخصيت خودشيفته، شامل الكوى فراكيرى

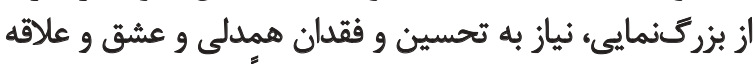

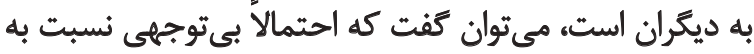

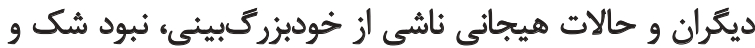

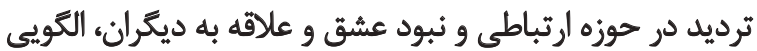

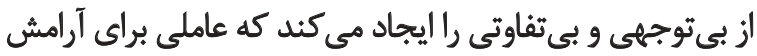

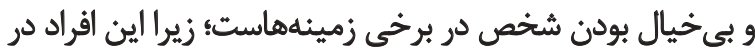

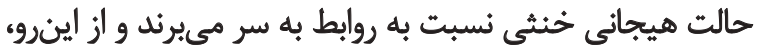

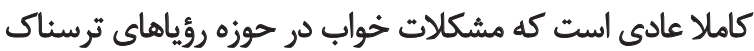

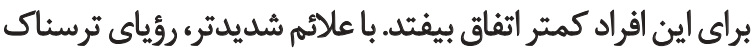
كمترى ديده مي ائود

در يُوهش حاضر نشان داده شده است كه بين شدت علائم

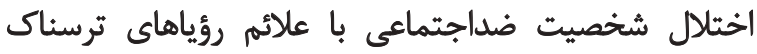

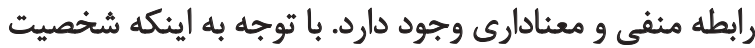

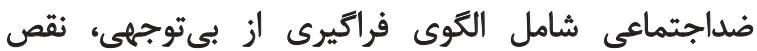

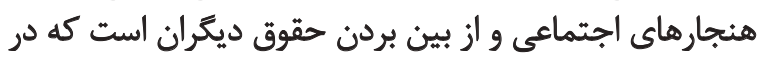

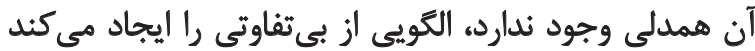

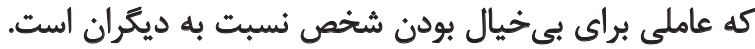

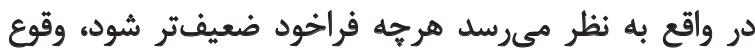

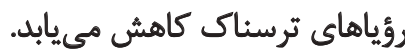

از سوى ديكر يك تبيين مهم مىتواند اين استدلال باشد كه
رابطه مثبت و معنادارى دارند. اختلال شخصيت خودشيفته و

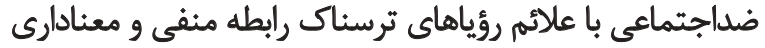

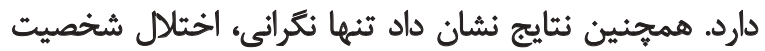

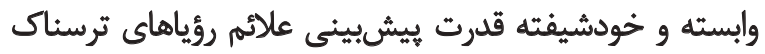

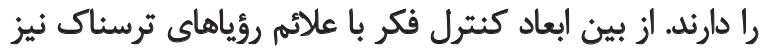

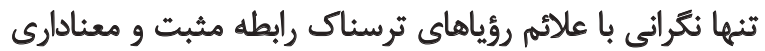

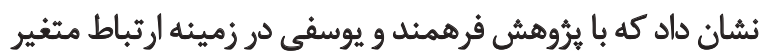

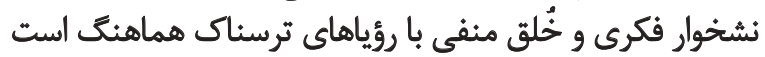

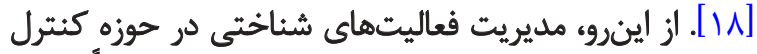

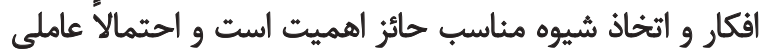

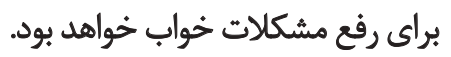

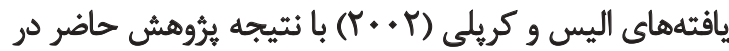

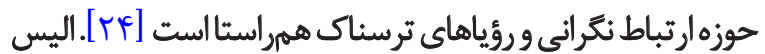

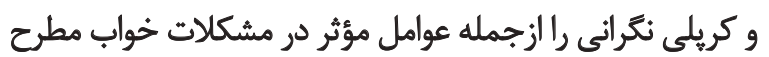

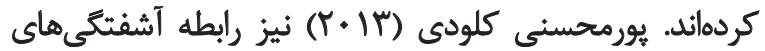

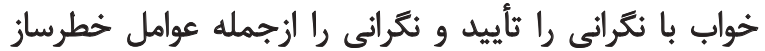

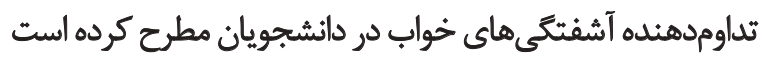

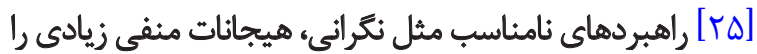

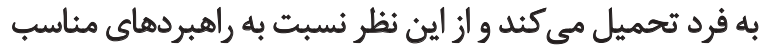

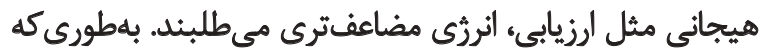

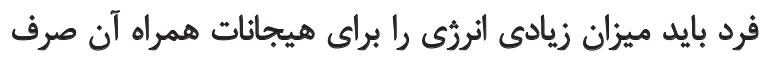

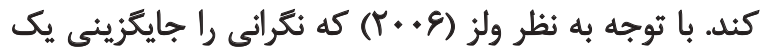

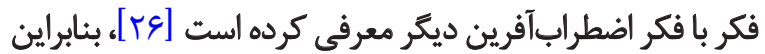

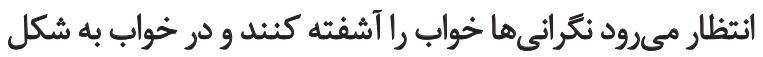
رؤياهاى ترسناك بروز يابند.

نتايج يثوهش حاضر نشان داد بين انواعى از اختلالات شخصيت شامل اختلال شخصيت وابسته و نمايشى باني بان علائم رؤياهاتى

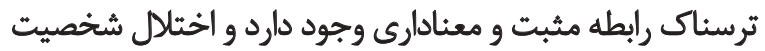

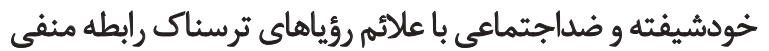

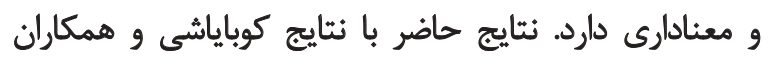
(Y. . V)

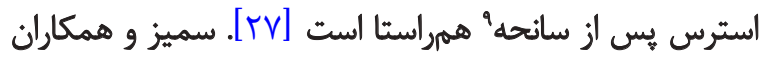

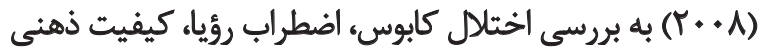

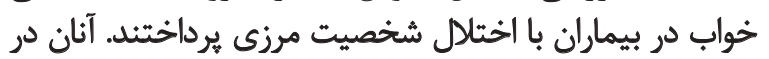

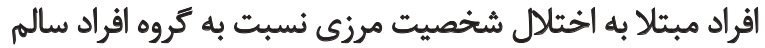

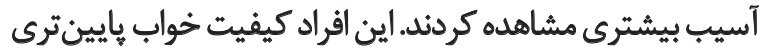

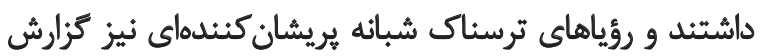

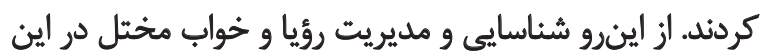

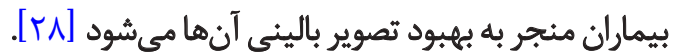
تبيين احتمالى در حوزه ارتباط اختلال شخصيت وابسته و رؤياهاى ترسناى آن است كه التوى شخصيت وابسته با بانياز 


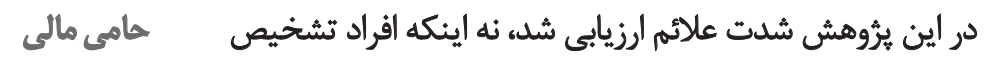
اين مطالعه با ثأييد دانشكده علوم تربيتى و روانشناسى آنادي

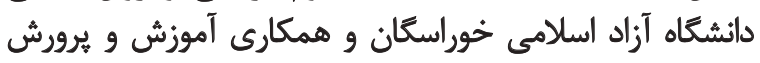
شهرضا و بدون حمايت مالى انجام شده است.

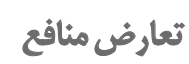

نويسندكان با يكديكر تضاد منافع نداشتند.

$$
\text { سياسُّزَإىى }
$$

از همكارى تمام افراد شركت كنئده قدردانى مىشود.

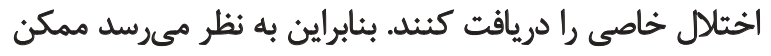

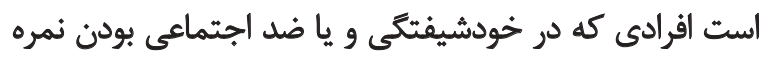

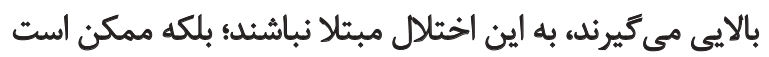

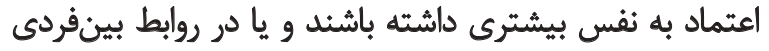

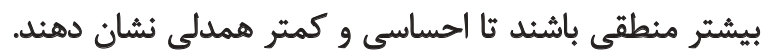

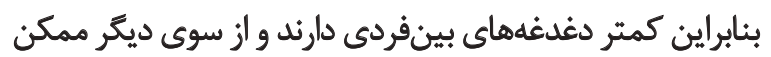

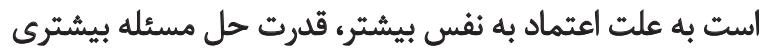

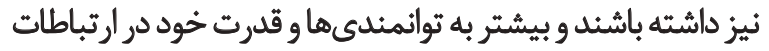

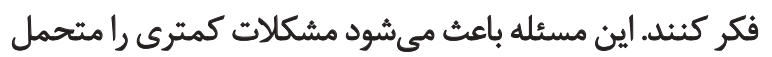
شوند و درنتيجه رؤياهاى ترسناك كمترى داشته باعث باشند.

\section{نتيجهَيرى}

با توجه به اين نتايج مى توان نتيجه كرفت شدت علائم اختلال

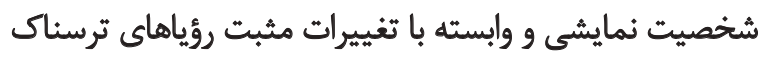

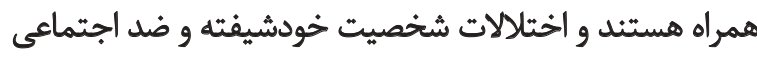

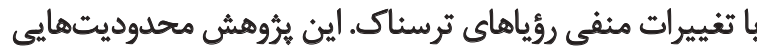

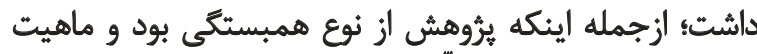

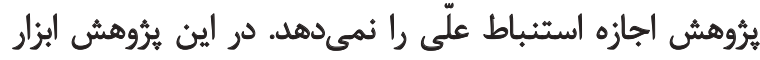

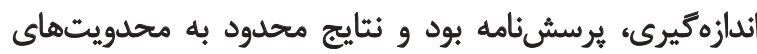

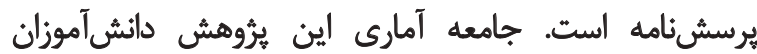

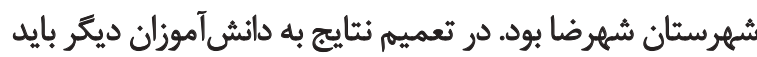

$$
\text { جانب احتياط رارعايت شرضان بود درد تعمين }
$$

با توجه به اين يافتهها از يك سو ونظر به شكل ثيرى شخصيت

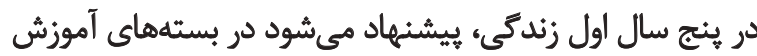

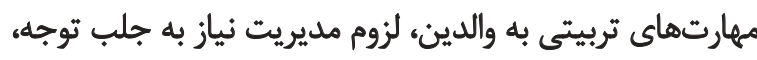

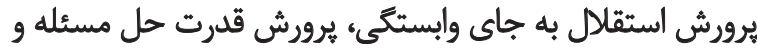

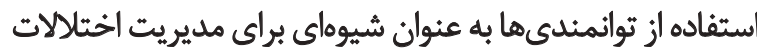

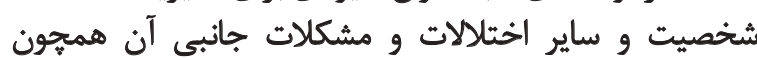

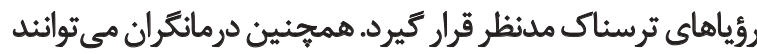

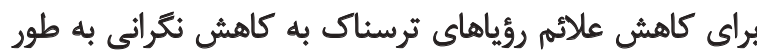

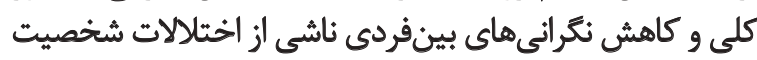
به عنوان يك مهم توجه داشته باشند. بكني

$$
\begin{aligned}
& \text { مالاحظات اخلاقى }
\end{aligned}
$$

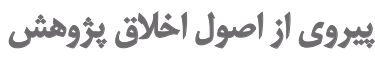

$$
\begin{aligned}
& \text { در اين يُروهش براى رعايت اخلاق يثروهشى شش نكته رعايت }
\end{aligned}
$$

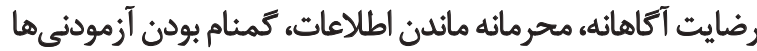

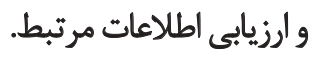

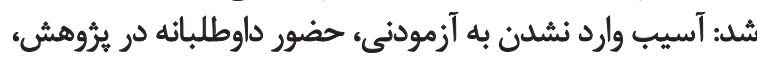




\section{References}

[1] Payne JD, Kensinger EA. Stress, sleep, and the selective consolidation of emotional memories. Current Opinion in Behavioral Sciences. 2018; 19(1):36-43. [DOI:10.1016/j.cobeha.2017.09.006]

[2] Rosen M. Sleep and dreaming. New York: Infobase Publishing; 2006.

[3] Sholtz D, Sholtz S. Personality theories [Y. Seyed Mohammadi, Persian trans]. Tehran: Virayesh; 2006.

[4] Harvey AG. I can't sleep, my mind is racing! An investigation of strategies of thought control in insomnia. Behavioural and Cognitive Psychotherapy. 2001; 29:3-11. [DOI:10.1017/ S1352465801001023

[5] Holmes EA, Brown RJ, Mansell W, Fearon R, Hunter EC, Frasquilho F, et al. Are there two qualitatively distinct forms of dissociation? A review and some clinical implications. Clinical Psychology Review. 2005; 25:1-23. [DOI:10.1016/j. cpr.2004.08.006] [PMID]

[6] Belicki D, Belicki K. Nightmares in a university population. Sleep Research. 1982; 11:116.

[7] Nielsen TA, Stenstrom P, Levin R. Nightmare frequency as a function of age, gender, an September 11, 2001: Findings from an internet questionnaire. Dreaming. 2006; 16(3):145-58. [DOI:10.1037/1053-0797.16.3.145]

[8] Levin R, Fireman G. Nightmare prevalence, nightmare distress, and self-reported psychological disturbance. Sleep. 2002; 25:205-21. [PMID]

[9] American Psychiatric Association. Diagnostic and statistical manual of mental disorders. Washington: American Psychiatric Association; 2013.

[10] Wells A, Davies MI. The Thought Control Questionnaire: a measure of individual differences in the control of unwanted thoughts. Behaviour Research and Therapy. 1994; 32(8):871-8. [DOI:10.1016/0005-7967(94)90168-6]

[11] Khanipor H, Sohrabi F, Tabatabaei S. [Compare beliefs and thought control strategies in normal and pathological levels of concern for students (Persian)]. Clinical Psychology Research and Consulting. 2011;1(1):71-82. [DOI: 10.22067/ijap.v1i1.2138]

[12] Wells A, Cartwright-Hatton H. A short form of the metacognitions questionnaire: properties of the MCQ-30. Behaviour Research and Therapy. 2004; 42(4):385-96. [DOI:10.1016/S00057967(03)00147-5]

[13] Agargun MY, Kara H, Ozer OA, Selvi Y, Kiran U, Kiran S. Nightmares and dissociative experiences: The key role of childhood traumatic events. Psychiatry and Clinical Neuroscience. 2003; 57:139-45. [DOI:10.1046/j.1440-1819.2003.01093.x] [PMID]

[14] Moo-Estrella J, Perez-Benitez H, Solis-Rodriguez F, Arandowsky- Sandoval G. Evaluation of depressive symptoms and sleep alterations in college students. Archives of Medical Research. 2005; 36(4):393-98. [DOI:10.1016/j.arce med.2005.03.018] [PMID]

[15] El-Sheikh M, Kelly RJ, Buckhalt JA, Benjamin Hinnant J. Children's sleep and adjustment over time: the role of socioeconomic context. Child. 2010; 81(3):870-83. [DOI:10.1111/j.14678624.2010.01439.x]
[16] Wong MM, Brower KJ, Nigg JT, Zucker RA. Childhood sleep problems, response inhibition, and alcohol and drug outcomes in adolescence and young adulthood. Alcoholism: Clinical and Experimental Research. 2010; 34(6):1033-44. [DOI:10.1111/j.15300277.2010.01178.x] [PMID] [PMCID]

[17] Fricke-Oerkermann L, Pluck J, Schredl M, Heinz K, Mitschke A, Waiter A, et al. Prevalence and course of sleep problems in childhood. Sleep. 2007; 30(10):1371-77. [DOI:10.1093/sleep/30.10.1371] [PMID] [PMCID]

[18] Farahmand Dehghanpoor F, Yousefi. [Multiple relationships between rumination and mood with frightening dreams (Persian)]. Iranian Journal of Psychiatry and Clinical Psychology. 2016; 22(2):92-102.

[19] Tabachnick BG, Fidell LS. Using Multivariate Statistics. $6^{\text {th }}$ edition. Boston: Pearson; 2013.

[20] Dabagh Z, Yousefi Z. [Reliability and validity of nightmare scale among girl adolescents (Persian)]. (nd). Under Publishing.

[21] Scarpa A, Wilson LC, Wells AO, Patriquin MA, Tanaka A. Thought control strategies as mediators of trauma symptoms in young women with histories of child sexual abuse. Behaviour research and therapy. 2009; 47(9):809-13. [DOI:10.1016/j. brat.2009.06.002] [PMID]

[22] Fata L, Moutabi, F, Moloudi R, Ziyaei k. [Psychometric properties of Persian version of thought control questionnaire and anxious thought inventory in Iranian students (Persian)]. Journal of Psychological Methods and Models. 2010; 1(1):81-103.

[23] Millon T, Millon c, Davis R, Grossman S. Millon Clinical Multiaxial Inventory-III. Bloomington: Pearson Assessments; 1997.

[24] Ellis J, Cropley M. An examination of thought control strategies employed by acute and chronic insomniacs. Sleep Medicine. 2002; 3:393-400. [DOI:10.1016/S1389-9457(02)00039-4]

[25] Pormohseni Kelori F. [The impact of rumination and worry on sleep disturbance (Persian)]. Daneshvar Medicine. 2014; 21(108):29-40

[26] Davey GCL, Wells A. The metacognitive model of worry and generalised anxiety disorder. Worry and its psychological disorders. 2006; 22:179-99. [DOI:10.1002/9780470713143.ch11]

[27] Kobayashi H, Boarts J, Delahanty D. Polysomnographically measured sleep abnormalities in PTSD: A meta-analytic review. Psychophysiology. 2007; 44(4):660-669. [DOI:10.1111/j.14698986.2007.537.x] [PMID]

[28] Semiz UB, Basoglu C, Ebrinc S, Cetin M. Nightmare disorder, dream anxiety, and subjective sleep quality in patients with borderline personality disorder. Psychiatry and Clinical Neuroscience. 2008; 62(1):48-5. [DOI:10.1111/j.14401819.2007.01789.x] [PMID] 